\title{
A Stackelberg Game for Incentive Proactive Caching Mechanisms in Wireless Networks
}

\author{
Fei Shen ${ }^{\diamond}$ Kenza Hamidouche ${ }^{\diamond}$, Ejder Baştug ${ }^{\dagger}, \diamond$ and Mérouane Debbah ${ }^{\diamond, \circ}$ \\ ${ }^{\diamond}$ Large Networks and Systems Group (LANEAS), CentraleSupélec, \\ Université Paris-Saclay, 3 rue Joliot-Curie, 91192 Gif-sur-Yvette, France \\ ${ }^{\dagger}$ Research Laboratory of Electronics, Massachusetts Institute of Technology, \\ 77 Massachusetts Avenue, Cambridge, MA 02139, USA \\ ${ }^{\circ}$ Mathematical and Algorithmic Sciences Lab, Huawei France R\&D, Paris, France \\ \{fei.shen, kenza.hamidouche\}@ centralesupelec.fr, ejder@mit.edu, merouane.debbah@ huawei.com
}

\begin{abstract}
In this paper, an incentive proactive cache mechanism in cache-enabled small cell networks (SCNs) is proposed, in order to motivate the content providers (CPs) to participate in the caching procedure. A network composed of a single mobile network operator (MNO) and multiple CPs is considered. The MNO aims to define the price it charges the CPs to maximize its revenue while the CPs compete to determine the number of files they cache at the MNO's small base stations (SBSs) to improve the quality of service $(\mathrm{QoS})$ of their users. This problem is formulated as a Stackelberg game where a single MNO is considered as the leader and the multiple CPs willing to cache files are the followers. The followers game is modeled as a noncooperative game and both the existence and uniqueness of a Nash equilibrium (NE) are proved. The closed-form expression of the NE which corresponds to the amount of storage each $\mathrm{CP}$ requests from the MNO is derived. An optimization problem is formulated at the MNO side to determine the optimal price that the MNO should charge the CPs. Simulation results show that at the equilibrium, the MNO and CPs can all achieve a utility that is up to $50 \%$ higher than the cases in which the prices and storage quantities are requested arbitrarily.
\end{abstract}

\section{INTRODUCTION}

The ever increasing number of mobile phones and connected devices is expected to contribute to an $800 \%$ increase in mobile data traffic in the five upcoming years [1]. Bandwidth intensive applications such as video-on-demand traffic will represent more than $70 \%$ of the global generated data requests [1]. To support this growing traffic and offload macro base stations, short range small base stations (SBSs) are deployed closer to the end-users. However, these SBSs are connected to a core network via capacity-limited backhaul links which makes it difficult to meet users' requirements in terms of quality-ofservice (QoS), especially during peak hours. To deal with this problem, distributed caching at the network edge has recently been proposed as a promising solution [2].

The idea of distributed caching consists in equipping the SBSs with storage units in which files are cached according to a placement policy. Thus, the SBSs can serve most of the requests locally without using the backhaul. However, for a successful deployment of proactive edge caching, the mobile network operators (MNOs) require cooperation of the content providers (CPs) to be able to cache their content at the SBSs [3]. To this end, incentive mechanisms must be developed by

This research has been supported by the ERC Starting Grant 305123 MORE (Advanced Mathematical Tools for Complex Network Engineering), the U.S NSF Grant CCF-1409228, and the projects 4GinVitro and BESTCOM. the operators to incite CPs to share and cache their content. The MNO offers to the CPs the caching service that allows the CPs' users to improve their QoS and in return the CPs pay a price defined by the MNO depending on the amount of storage space requested by each CP.

Recently, several caching works have appeared from different aspects, such as optimal caching policies in layered video delivery [4], device-to-device (D2D) networks [5], hieararchical caching [6], multi-cell scenario with limitedbackhaul [7] and so on (see [3] for detailed discussions). More relevantly, there exist some works focusing on economic aspect of caching in wireless D2D networks. In such networks, the operators define pricing scheme to motivate users to proactively download the most popular files and cache them in their devices to serve other users' requests. In [8], the authors proposed a smart pricing scheme to maximize the benefit of the operator and minimize the charged price to the users. Via D2D communications, users can trade their cached files to minimize their expected payments. On the other hand, the operator defines a dynamic pricing model that differentiates off-peak and peak time periods to maximize its own benefit. The authors in [9] formulated the cache incentive problem as a Stackelberg game in which the SBSs are the leaders and the users are the followers. In this model, the SBSs start by anticipating users' reactions and determine the optimal price that maximizes their offloaded traffic from to the users devices. Given a defined reward by the SBSs, users can then decide whether to help the SBSs by caching the files and serving other users or not.

Despite being interesting, all these works focused on cache incentive mechanisms in D2D wireless networks and ignored the role of CPs in the caching process. The main contribution of this work is to propose a new cache incentive mechanism between an MNO and multiple CPs. We formulate the cache incentive problem as a Stackleberg game in which the MNO is a leader and the CPs are the followers. The MNO predicts the requests profile of the CPs and define the price that maximizes its revenue. On the other hand, due to limited storage capacity of the SBSs, CPs cannot cache all their files, thus, the CPs compete to maximize the amount of storage space they request given the fixed price by the MNO. The competition between the CPs is formulated as a non-cooperative sub-game in which each $\mathrm{CP}$ aims to maximize the satisfaction of its users in terms of transmission rate. Both the existence and uniqueness of the 


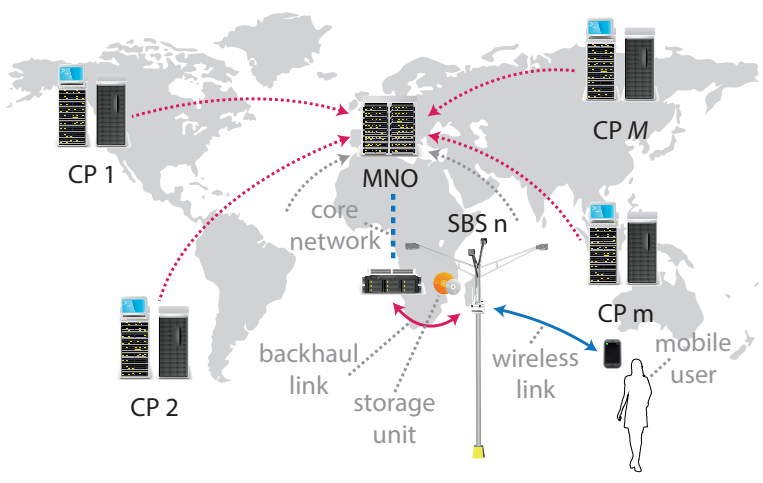

Figure 1: An illustration of the system model.

Nash equilibrium (NE) are proved. The NE represents a state in which none of the CPs can improve its benefit by requesting a different amount of storage space given the requested amount by the other CPs fixed. We provide closed-form expressions of the storage amount the CPs must request at the NE. Then, given the request profiles of the CPs, an optimization problem is formulated at the MNO to determine the optimal price that should be charged to the CPs to maximize the MNO's revenue. Simulation results show that the MNO can get a revenue that is up to $50 \%$ higher compared to the case in which the prices are selected arbitrarily. Moreover, at the equilibrium, we show that the CPs can achieve a utility that is $20 \%$ to $50 \%$ higher compared to the two cases in which half and the double of the storage space at the NE are requested by the CPs, respectively.

The rest of the paper is organized as follows. In Section III. we define the system and the caching models. The Stackelberg game is formulated in Section III, and the analysis is conducted in Section IV The numerical results are presented in Section V. We finally draw conclusions in Section VI.

\section{PRELiminaries}

\section{A. System Model}

We consider an heterogeneous network (HetNet) which consists of $N$ cache-enabled SBSs and is controlled by one MNO. There are $M$ CPs that are willing to cache their files in the HetNet in order to enhance the QoS of their users. Let $\mathcal{N}=\{1,2, \ldots, N\}$ denote the set of cacheenabled SBSs, each of which has a limited capacity $s_{n}$, and $\mathcal{M}=\{1,2, \ldots, M\}$ denote the set of CPs. Each CP requests from the MNO to cache its $q_{m}$ most popular files. The $N$ SBSs are connected to the MNO with a backhaul such as DSL, optical fibers or wireless backhaul. All the user equipments (UEs) communicate with the corresponding SBSs. If the desired files from the UEs are not cached in the SBSs, they are served by the MNO via the capacity-limited backhaul links. The system model is depicted in Fig. 1

Each CP $m$ has a local content catalog $\mathcal{F}_{m}$ with $F_{m}$ files. The global files catalog is denoted $\mathcal{F}=\bigcup \mathcal{F}_{m}$, and all the files are assumed to have the same unit size. We assume that each file $f_{i}$ is cached by the MNO $p_{f_{i}}$ times at the SBSs based on the popularity of the file. The goal of each CP is to cache its most popular files locally at the SBSs so that the users experience an improved data transmission rate. However, the
Table I: List of Notations.

\begin{tabular}{|l|l|}
\hline $\mathcal{N}=\{1,2, \ldots, N\}$ & set of SBSs \\
\hline $\mathcal{M}=\{1,2, \ldots, M\}$ & set of CPs \\
\hline$q_{m}$ & The number of files that CP $m$ wants to cache \\
\hline$p_{f_{i}}$ & The number of cached copies of file $f_{i}$ \\
\hline$s_{n}$ & The cache size of each SBS $n$ \\
\hline$\pi$ & The charged price for caching a given file \\
\hline$u_{o}$ & The utility of MNO \\
\hline$u_{m}$ & The utility of CPs \\
\hline $\mathcal{Q}_{m}$ & The set of files requested by each CP $m$ \\
\hline$p_{m}$ & The mean access probability of CP $m$ 's files \\
\hline$d\left(\mathcal{Q}_{m}\right)$ & The total caching copies of requested files of all CPs \\
\hline
\end{tabular}

storage capacity of the SBSs is limited and not all the files can be cached with sufficient copies. The notations used in the rest of this paper is summarized in Table I.

\section{B. Cache Incentive Model}

We formulate the cache incentive problem as a Stackelberg game in which a non-cooperative game is involved as a subgame. As shown in Fig. 1, the Stackelberg game is played between the MNO and the $M$ CPs. On the one hand, all the CPs wish to cache as many as possible files at the MNO's HetNet such that the QoS of their users (for example the delay) is improved. On the other hand, there exists a cost for caching. Therefore, the incentive proactive caching mechanism is controlled by a charge price determined by the MNO to optimize its revenue in the proposed Stackelberg game. The sequential game (Stackelberg) leads to a more competitive equilibrium than the simultaneous move game: The MNO plays as the leader providing the caching price $\pi$ to all the CPs, while the CPs play as the followers reacting with their optimal number of files they want to cache based on the given price. Both the MNO and the CPs are rational and optimize their own utilities denoted as $u_{o}$ and $u_{m}$, respectively. The strategy of the leader MNO is the caching price $\pi$ and the strategies of the followers CPs are their the number of files they want to cache $q_{m}(\pi)$. It should be noted that the price defined by the MNO depends on the used caching policy and the storage capacity of all the SBSs.

Due to the limited caching capacity, the $M$ CPs compete for the possible caching quantities. Therefore, a non-cooperative game is played as a sub-game among the $M$ CPs. We assume that the file access probability of each file is perfectly known at all the entities (i.e., CPs and MNO). The strategies of the CPs are the quantity of their caching requests. Under the perfect knowledge of the access probability of each caching file, the CPs will choose to cache the first $q_{m}^{*}$ files ordered with the highest access probability, i.e., popularity.

\section{Stackelberg Game Formulation}

In this section, we provide the utilities of the MNO and the CPs, respectively. The proposed Stackelberg game is played as follows. The leader plays first by providing its optimal 
strategy to all the followers after predicting the strategies of the followers. Then, the followers reply with their best strategies given the strategy of the leader. The result of the Stackelberg game is that both the leader and the followers optimize their utilities. The Stackelberg equilibrium is exactly the point that the leader wishes. The basic idea of the utilities for both the MNO and the CP can be formulated as a general function, such as Utility = Revenue - Cost. In the following, we will analyse the utilities of the MNO and the CPs, respectively.

\section{A. Utility of the $M N O$}

For the MNO, the main cost is the caching storage cost denoted as $C_{o}$. This caching storage cost is a function of the caching capacity of all the SBSs and the number of times each file is cached. Therefore, the cost of caching all CPs files $C_{o}$ for the MNO can be defined as the following barrier function:

$$
C_{o}= \begin{cases}\frac{1}{S-d\left(\mathcal{Q}_{\mathcal{M}}\right)} & \text { if } 0<d\left(\mathcal{Q}_{\mathcal{M}}\right) \leq S \\ \infty & \text { otherwise, }\end{cases}
$$

where $d\left(\mathcal{Q}_{\mathcal{M}}\right)$ is the amount of all the cached files by the $\mathrm{MNO}$ and is given by:

$$
d\left(\mathcal{Q}_{\mathcal{M}}\right)=\sum_{m=1}^{M} \sum_{f_{i} \in \mathcal{Q}_{m}} p_{f_{i}}
$$

where $p_{f_{i}}$ is the number of copies of file $f_{i}$ that are cached at the SBSs and defined by the MNO depending on the popularity of file $f_{i}$. The caching capacity of the MNO is given by:

$$
S=\sum_{n=1}^{N} s_{n}
$$

The revenue of the MNO in the caching problem is the total charge from all the CPs for caching their files. The price of caching one file is denoted by $\pi$ and the number of files that is requested by a $\mathrm{CP} m$ to be cached is $q_{m}$. Thus, the total revenue of the $\mathrm{MNO}$ can be given by

$$
R_{o}=\pi \sum_{m=1}^{M} q_{m}(\pi)
$$

Now we obtain the utility function of the MNO in our proposed Stackelberg game as a function of the quantity of caching request from all the CPs, $\mathbf{q}=\left[q_{1}, \ldots, q_{M}\right]$.

$$
u_{o}(\mathbf{q}(\pi))=R_{o}(\mathbf{q}(\pi))-C_{o}(\mathbf{q}(\pi)),
$$

where $R_{o}$ and $C_{o}$ are defined in (1) and (4), respectively.

\section{B. Utility of the CPS}

Given that the MNO fixes a price $\pi$ for a storage unit, all the CPs reply with their quantity of caching request by optimizing their own utilities. We assume that each CP is independent of the others and makes its best strategy only based on its local information.

The cost of CPs for the caching requests are the charge paid to the MNO for their desired quantity of caching files. For each CP $m$, the cost $C_{m}$ is defined as

$$
C_{m}=\pi q_{m}(\pi)
$$

where $q_{m}(\pi)$ is a function of the provided charge price $\pi . C_{m}$ is an increasing function of the caching quantity $q_{m}(\pi)$.

In fact, the CPs such as Facebook and Youtube are concerned about the satisfaction of their users. This user satisfaction, which can be the delay of downloading a file or the data throughput, is an increasing function of the caching request quantity $q_{m}$ of $\mathrm{CP} m$ and a decreasing function of the caching request quantities $\mathbf{q}_{-m}$ of others because of the limited caching capacity. $\mathbf{q}_{-m}$ is defined as the caching request quantities of all the other CPs except $\mathrm{CP} m$, i.e.,

$$
\mathbf{q}_{-m}=\left[q_{1}, \ldots, q_{m-1}, q_{m+1}, \ldots, q_{M}\right] .
$$

The revenue of CPs can be easily defined as the satisfaction function of each $\mathrm{CP}$. We assume that the $q_{m}$ files with the highest access probabilities in each $\mathrm{CP} m$ are requested. Then the satisfaction function of CP $m$ is as follows, which is an increasing function of the caching request quantity.

$$
R_{m}=\log \left(1+\frac{q_{m}}{1+\frac{1}{\alpha_{m}} J_{m}}\right),
$$

where $J_{m}=\sum_{l \neq m} q_{l}$ is the quantity of caching files requested by all the other CPs except CP $m$ and $\alpha_{m}$ is the amount of generated requests by the users of $\mathrm{CP} m$. The satisfaction $R_{m}$ of a CP $m$ was chosen as it is an increasing function of the number of files that are cached by a CP $m$ as well as its traffic load $\alpha_{m}$. Indeed, the more files are cached at the SBSs and the higher the popularity of the files, the higher is the number of requests that can be served locally from the SBSs. Thus, more users of $\mathrm{CP} m$ can experience a higher transmission rate. On the other hand, the satisfaction of a CP $m$ decreases as the number of files cached by the other CPs increases. In this case, less storage is available for CP $m$ and a higher number of its requests need to be served through the backhaul. It should be noted that any other function that has the same properties as $R_{m}$ can be chosen as a satisfaction function.

Given the constraints on the caching capacity of the mobile network, now we obtain the utility function of each $\mathrm{CP} m$ as

$$
u_{m}=R_{m}-C_{m}
$$

where the cost $C_{m}$ and the revenue $R_{m}$ are defined in (6) and (8), respectively. It is obvious that $u_{m}$ is a concave function of the quantity of the caching requests.

Given the charge price from the MNO, all the CPs respond with their quantities of caching requests simultaneously by maximizing their own utilities. Due to the limited caching capacity $S$, all the CPs can be considered as competitive players competing for the caching quantities. Therefore, we can formulate the quantity optimization problem of CPs as a non-cooperative game $\mathcal{G}=\mathcal{G}(\mathcal{M}, \mathcal{Q}, \mathcal{U})$ consisting of the following components:

- The set of players in $\mathcal{G}$ is the set $\mathcal{M}$ of CPs.

- Given the quantity profile $\mathbf{q}_{-m} \in \mathcal{Q}_{m}=\prod_{l \neq m} \mathcal{Q}_{l}$ of the opponents of player $m$, the feasible action set of CP in the presence of the caching capacity $d\left(\mathcal{Q}_{M}\right) \leq S$ is

$$
\mathcal{Q}_{M}\left(\mathbf{q}_{-m}\right)=\left\{\mathbf{q}_{m} \in \mathcal{Q}_{m}: \mathbf{q}_{m} \geq 0\right\} .
$$


- The utility $u_{m}$ of player $m$ is given by 9 .

In this context, the most adopted solution concept is the NE.

Definition 1. A quantity profile $\mathbf{q}^{*}$ is a NE of the caching request quantity game $\mathcal{G}$ if

$$
\begin{array}{cc}
\mathbf{q}_{m}^{*} \in \mathcal{Q}_{m}\left(\mathbf{q}_{-m}^{*}\right), & \forall m \in \mathcal{M}, \\
u_{m}\left(\mathbf{q}^{*}\right) \geq u_{m}\left(\mathbf{q}_{m}, \mathbf{q}_{-m}^{*}\right), & \forall \mathbf{q}_{m} \in \mathcal{Q}_{m}\left(\mathbf{q}_{-m}^{*}\right) .
\end{array}
$$

Provided the utility functions for the MNO and CPs, the optimal strategies of the leader and the followers are derived in the next section. In the Stackelberg game, the followers' strategies are predicted before the leader makes its own strategy. Therefore, the optimal quantity of caching requests of the CPs is analyzed first.

\section{Stackelberg Game Solution}

In this section, we derive the solution of the proposed Stackelberg game. In the sequential (Stackelberg) game, the leader moves first by predicting the strategies of the followers. The followers reply by optimizing their own utilities given the strategy of the leader. The result of the game is that the followers play exactly what the leader wishes. Therefore, the optimal strategy of the followers should be predicted first. In the following, we obtain the optimal quantities of caching requests of the CPs in the closed form.

\section{A. Optimal Quantity of Caching Request}

The CPs optimize their strategies of the quantity of the caching requests and provide them to the MNO given the charge price $\pi$ announced by the MNO. The optimal $q_{m}$ is obtained by solving the following problem.

$$
q_{m}=\arg \max _{\mathbf{q}} u_{m} \quad \text { subject to } \quad \mathbf{q} \geq 0 .
$$

Given the charge price for the caching files, the CPs compete for the quantity of caching requests, which formulates a noncooperative game. In the following, we find the solution for this non-cooperative game.

Proposition 1 (Best Response). Given the charge price $\pi$ announced by the MNO, the best response (BR) of each CP is the quantity of caching files it requests, which is

$$
q_{m}^{B R}=\left(\frac{1}{\pi}-1-\frac{J_{m}}{\alpha_{m}}\right)^{+},
$$

with $\alpha_{m} \geq M$.

Proof: The BR of the caching request quantity $q_{m}^{B R}$ is obtained by checking the first derivative of $u_{m}$ with respect to $q_{m}$,

$$
\frac{\partial u_{m}}{\partial q_{m}}=\frac{\alpha_{m}}{\alpha_{m}+\alpha_{m} q_{m}+J_{m}}-\pi=0 .
$$

The second derivative of $u_{m}$ with respect to $q_{m}$ is

$$
\frac{\partial^{2} u_{m}}{\partial q_{m}^{2}}=\frac{-\alpha_{m}^{2}}{\left(\alpha_{m}+\alpha_{m} q_{m}+J_{m}\right)^{2}}<0
$$

which guarantees a global optimal of $u_{m}$. Since the utility of each CP $u_{m}$ is a convex function of $q_{m}$, 14 is proved. The function ()$^{+}$is to ensure the requested caching quantity to be a non-negative value.

Remark 1. The total quantity of the caching requests of all the other CPs can be learnt or fed back at each CP. The $q_{m}^{B R}$ is based on the local information $\alpha_{m}, q_{m}$ and the feedback $J_{m}$ as a single value of the summation.

Now we prove that the proposed non-cooperative game admits a unique NE.

Theorem 1 (Nash Equilibrium). Given the charge price $\pi$ announced by the MNO, the NE of each $C P$ is the quantity of caching files it requests, which is

$$
q_{m}^{N E}=\frac{D_{m}}{D}=\frac{\left(\frac{1}{\pi}-1\right) a_{m} b_{m}}{D},
$$

where $a_{m}$ is

$a_{m}= \begin{cases}1-\frac{1}{\alpha_{m}}-\frac{1-\alpha_{1}}{\alpha_{1}} \sum_{l=2, l \neq m}^{M} \frac{\alpha_{m}-\alpha_{l}}{\left(\alpha_{l}-1\right) \alpha_{m}} & \text { if } m \neq 1 \\ 1-\frac{1}{\alpha_{1}}-\frac{1-\alpha_{M}}{\alpha_{M}} \sum_{l=2}^{M-1} \frac{\alpha_{1}-\alpha_{l}}{\left(\alpha_{l}-1\right) \alpha_{1}} & \text { if } m=1\end{cases}$

and $b_{m}$ is

$$
b_{m}= \begin{cases}\prod_{l=2, l \neq m}^{M}\left(1-\frac{1}{\alpha_{l}}\right) & \text { if } m \neq 1 \\ \prod_{l=2}^{M-1}\left(1-\frac{1}{\alpha_{l}}\right) & \text { if } m=1\end{cases}
$$

and

$$
D=\left(1-\frac{1-\alpha_{1}}{\alpha_{1}} \sum_{l=2}^{M} \frac{1}{\alpha_{l}-1}\right) \prod_{l=2}^{M}\left(1-\frac{1}{\alpha_{l}}\right) .
$$

If $M=2$, then $\sum_{l=2}^{M-1} \frac{\alpha_{1}-\alpha_{l}}{\left(\alpha_{l}-1\right) \alpha_{1}}=0$ and $\prod_{l=0}^{M-1} 1-\frac{1}{\alpha_{l}}=1$.

Proof: We give only the sketch of the proof due to the space limitations. The trick for finding the NE of the proposed non-cooperative game is to jointly solve the $M$ functions of the BR for all the CPs. The $M$ functions of the BR can be formulated as a matrix function denoted as $\mathbf{D q}=\mathbf{C}$ where the matrix $\mathbf{D}$ and vectors $\mathbf{q}$ and $\mathbf{C}$ are as follows, respectively.

$$
\left[\begin{array}{cccc}
1 & \frac{1}{\alpha_{1}} & \cdots & \frac{1}{\alpha_{1}} \\
\frac{1}{\alpha_{2}} & 1 & \cdots & \frac{1}{\alpha_{2}} \\
\vdots & \vdots & \ddots & \vdots \\
\frac{1}{\alpha_{M}} & \frac{1}{\alpha_{M}} & \cdots & 1
\end{array}\right]\left[\begin{array}{c}
q_{1} \\
q_{2} \\
\vdots \\
q_{M}
\end{array}\right]=\left[\begin{array}{c}
\frac{1}{\pi}-1 \\
\frac{1}{\pi}-1 \\
\vdots \\
\frac{1}{\pi}-1
\end{array}\right] .
$$

The NE quantity of each $\mathrm{CP}$ is solved by applying the Cramer's rule $q_{m}=\frac{\operatorname{det}\left(\mathbf{D}_{m}\right)}{\operatorname{det}(\mathbf{D})}$, where $\operatorname{det}(\mathbf{D})$ is the determinant of matrix $\mathbf{D}$ and $\operatorname{det}\left(\mathbf{D}_{m}\right)$ is the determinant of matrix $\mathbf{D}_{m}$ which is formed by replacing the $m$-th column of $\mathbf{D}$ by the column vector $\mathbf{C}$.

Remark 2. In caching, there are two basic modes. One is coded caching in which the SBSs cache any number of bits from the files. In this case, the NE quantity derived in (17) is the actual quantity of files requested by $C P \mathrm{~m}$. The other one is uncoded caching, in which a given file can only be cached as a whole or equally divided chunks. In this case, the round function \lfloor\rceil is applied to $q_{m}^{N E}$ before $C P m$ replies to the $M N O$ in order to obtain the integer optimal quantity of caching request. In our model, we assume the uncoded caching case so that only integer quantity of caching requests are considered. 


\section{B. Optimal Charge Price}

After predicting the strategy policies of the CPs, the MNO optimizes the charge price $\pi$ by solving the following problem.

$$
\pi^{*}=\arg \max _{\pi} u_{o}(\mathbf{q}(\pi)) \quad \text { subject to } \pi \geq 0 .
$$

We observe from (1) and (2) that the quantity of caching request from each $\mathrm{CP} m$ exists in the size of the set $\mathcal{Q}_{m}$. Therefore, we assume that the number of copies of each file $f_{i} \in \mathcal{Q}_{m}$ that are cached are defined as the quantized vector given by:

$$
\mathbf{p}_{f_{i}}=\left[\ldots, p_{m}-\Delta p_{m}, p_{m}, p_{m}+\Delta p_{m}, \ldots\right]
$$

with size of $q_{m}$ for each $\mathrm{CP} m . p_{m}$ is the mean access probability of files requested by $\mathrm{CP} m$ and $\Delta p_{m}$ is the step size.

Given the number of times $\mathbf{p}_{f_{i}}$ each file $f_{i}$ is cached as defined in (23), we obtain

$$
\sum_{f_{i} \in \mathcal{Q}_{m}} p_{f_{i}}=q_{m}^{N E} f\left(p_{m}\right)
$$

where $f\left(p_{m}\right)$ is a function of the mean access probability $p_{m}$ with the following form

$$
f\left(p_{m}\right)= \begin{cases}p_{m} & \text { when } q_{m} \text { is odd, } \\ p_{m}+\frac{\Delta p_{m}}{2} & \text { when } q_{m} \text { is even. }\end{cases}
$$

Since the MNO can predict the NE quantities of all the CPs, which are functions of the charge price $\pi$, the utility function of the MNO is then

$$
u_{o}=\pi \sum_{m=1}^{M} q_{m}(\pi)-\frac{1}{S-\sum_{m=1}^{M} q_{m}(\pi) f\left(p_{m}\right)} .
$$

By predicting the requesting $q_{m}(\pi)$ from each $\mathrm{CP} m$, the MNO can make its own optimal strategy, which is the charge price $\pi^{*}$. Here we analyse the case where the total caching requests do not exceed the caching capacity of the HetNet.

Proposition 2 (Optimal Price). The optimal charge price $\pi^{*}$ provided by the MNO to maximize its own utility $u_{o}$ is as follows.

$$
\pi^{*}=\frac{\sqrt{\frac{r}{t}}+r}{S+r},
$$

where $r$ is a function of $\alpha$ and $t$ is a function of both $\alpha$ and $\mathbf{p}$ defined as

$$
t=\sum_{m=1}^{M} \frac{a_{m} b_{m}}{D}
$$

and

$$
r=\sum_{m=1}^{M} \frac{a_{m} b_{m} f\left(p_{m}\right)}{D} .
$$

Proof: Since $r$ and $t$ are independent of the optimization objective $\pi$, we rewrite the utility function of MNO $u_{o}$ as

$$
u_{o}=(1-\pi) t-\frac{1}{S-\left(\frac{1}{\pi}-1\right) r} .
$$

Then the first derivative with respect to $\pi$ is

$$
\frac{\partial u_{o}}{\partial \pi}=\frac{r}{(S \pi-(1-\pi) r)^{2}}-t
$$

The second derivative with respect to $\pi$ is

$$
\frac{\partial^{2} u_{o}}{\partial \pi^{2}}=\frac{-2 r(S \pi-(1-\pi) r)(S+r)}{(S \pi-(1-\pi) r)^{4}} .
$$

By observing that the MNO will definitely provide a price in order to ensure $d\left(\mathcal{Q}_{m}\right) \leq S, S \pi-(1-\pi) r>0$ and then $\frac{\partial^{2} u_{o}}{\partial \pi^{2}}<0$, which guarantees a global maximum $\pi^{*}$.

By solving $\frac{\partial u_{o}}{\partial \pi}=0$ in 31 , we get the value of $\pi^{*}$ in 27 , which completes the proof.

Notice that the charge price should be a positive value $\pi^{*}>$ 0 , therefore the result $\frac{-\sqrt{\frac{r}{t}}+r}{S+r}$ is dropped.

To ensure a positive total charge and also that the total caching requests do not exceed the caching capacity $S$, i.e., the cost $C_{o}$ is a positive limited value, the charge price given by the MNO should be restricted in the following range.

Corollary 1. The caching capacity $S$ of the HetNet can be fully exploited if the charge price given by the MNO follows the range

$$
\frac{r}{S+r}<\pi<1 \text {. }
$$

Proof: $\pi<1$ is proved by ensuring $\frac{1}{\pi}-1>0$ in 30 . $\frac{r}{S+r}<\pi$ is proved by ensuring $\frac{1}{S-\left(\frac{1}{\pi}-1\right) r}>0$ in 30 .

Remark 3. The optimal price $\pi^{*}$ provided in (27) always satisfies the feasible price range in (33).

\section{Example}

In this part, we show the 2-CP case as an example to illustrate the incentive Stackelberg game on caching. The protocol of the proposed Stackelberg game played between the MNO and CPs and the non-cooperative game played among the CPs are described as follows.

- The MNO predicts the NE quantities of the caching requests from all the CPs before making its own strategy.

$$
\begin{aligned}
& q_{1}^{N E}=\frac{\left(\frac{1}{\pi}\right)\left(\alpha_{1}-1\right) \alpha_{2}}{\alpha_{1} \alpha_{2}-1}, \\
& q_{2}^{N E}=\frac{\left(\frac{1}{\pi}\right)\left(\alpha_{2}-1\right) \alpha_{1}}{\alpha_{1} \alpha_{2}-1} .
\end{aligned}
$$

- The optimal charge price $\pi^{*}$ is the strategy of the MNO by maximizing its utility $u_{o}$.

$$
\pi^{*}=\frac{r+\sqrt{\frac{r}{t}}}{r+S},
$$

where $r=\sum_{m=1}^{2} \frac{\left(\alpha_{m}-1\right) \alpha_{l \neq m}}{\alpha_{1} \alpha_{2}-1}, \quad t=$ $\sum_{m=1}^{2} \frac{\left(\alpha_{m}-1\right) \alpha_{l \neq m}}{\alpha_{1} \alpha_{2}-1} f\left(p_{m}\right)$ and $f\left(p_{m}\right)=p_{m}+\frac{\Delta p_{m}}{2}$.

- Given the charge price for each file of the caching request, each CP chooses a quantity of caching files as its best response.

$$
\begin{aligned}
& q_{1}^{B R}=\left(\frac{1}{\pi}-1\right)-\frac{q_{2}}{\alpha_{1}} \\
& q_{2}^{B R}=\left(\frac{1}{\pi}-1\right)-\frac{q_{1}}{\alpha_{2}} .
\end{aligned}
$$




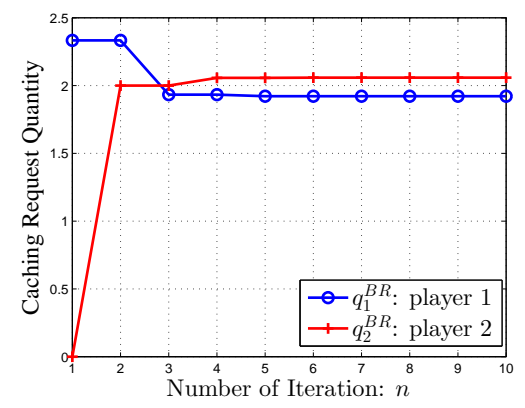

(a)

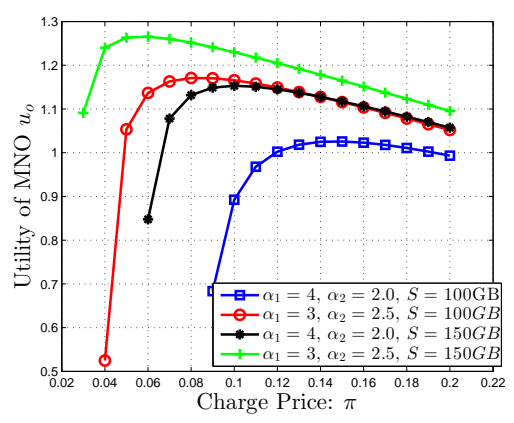

(b)

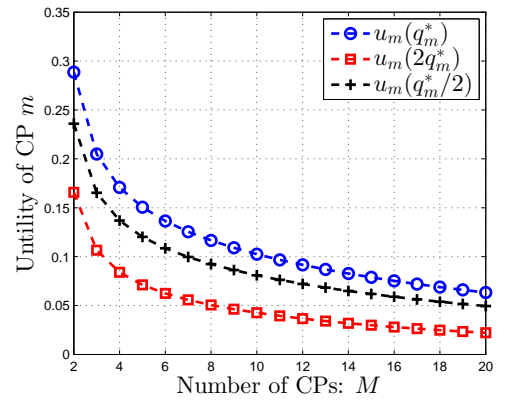

(c)

Figure 2: Numerical results for a) convergence of the best response for 2-CP case, b) utility function of the MNO with respect to charge price $\pi$ for 2-CP case, and c) utility of CP with respect to the total number of CPs $M$.

- The BR quantities of both CPs converge to the NE quantities in (34) and (35), respectively. The resulting $q_{m}^{N E}$ maximizes both the utilities of the MNO and CPs.

\section{NUMERICAL RESULTS}

In order to illustrate the outcome of the proposed gametheoretical cache problem, without loss of generality, we simulate the scenario of one MNO and $2 \mathrm{CPs}$ with different parameters. We assume identical file size as $10 G B$ for high quality videos.

Fig. 2a shows the BR dynamic of the non-cooperative game for the 2-CP case. The parameters of the simulations are as follows: $\alpha_{1}=5, \alpha_{2}=7$. The charge price provided by the MNO is set as $\pi=0.3$. The initial value of CP 2 is set to be $q_{2}^{\text {int }}=0$. We can observe that the proposed noncooperative game converges very rapidly. The convergence is irrespective of the initial points. The convergence values result in the same quantities as the theoretical NE solutions given in 17) in Thereom 1.

Fig. 2b shows the utility function of the MNO with respect to the charge price. We can see that the proposed utility of the MNO always admits a global optimum for different sets of parameters. The utilities of the MNO with higher caching capacity are higher. This is because more caching requests can be served and less caching cost is spent for the same amount of caching files. The difference of the starting points of the curves are due to the feasible price region in Corollary 1 The global optimum in Fig. $2 \mathrm{~b}$ result in the same price derived in 27). With the optimum price, the utility of the MNO improves even up to $50 \%$ than arbitrary chosen prices.

Fig. 2c shows the utility of a single $\mathrm{CP}$ with respect to the total number of CPs $M$ while the quantity of the caching request $q_{m}$ changes. The charge price is provided as the optimal price $\pi^{*}$ for different total number of CPs. The optimal quantity $q_{m}^{*}$ is derived accordingly. The utilities with double quantity $2 q_{m}^{*}$ and half quantity $\frac{q_{m}^{*}}{2}$ are provided for comparison, respectively. From Fig. 2c, we observe that the more CPs, the lower the utility $u_{m}$ of each CP. This is due to the increasing number of CPs which results in the increase of the amount of storage space that is requested by the CPs and thus, a higher price is charged by the MNO. Moreover, the utility of each $\mathrm{CP}$ decreases according to (8) when the total storage capacity allocated for the other CPs increases. We can also see that by requesting the optimal caching quantity $q_{m}^{*}$, each CP achieves $20 \%$ higher utility than requesting $\frac{q_{m}^{*}}{2}$ and up to $50 \%$ than requesting $2 q_{m}^{*}$.

\section{CONCLUSIONS}

We have studied a Stackelberg game of proactive edge caching between the leader MNO and the followers CPs. The best response, the resulting NE caching quantities, and the optimum charge price have been derived in closed forms; and the convergence of our proposed incentive mechanism has been validated via numerical studies. These numerical results also showed that both the MNO and CPs can achieve up to $50 \%$ higher utilities in the proposed Stackelberg game. This clearly points out the need of incentive caching mechanisms in $5 \mathrm{G}$ wireless networks.

\section{REFERENCES}

[1] Cisco, "Cisco visual networking index: Global mobile data traffic forecast update, 2015-2020," White Paper, 2016.

[2] E. Baştuğ, M. Bennis, and M. Debbah, "Living on the Edge: The role of proactive caching in $5 \mathrm{G}$ wireless networks," IEEE Communications Magazine, vol. 52, no. 8, pp. 82-89, August 2014.

[3] G. Paschos, E. Baştuğ, I. Land, G. Caire, and M. Debbah, "Wireless caching: Technical misconceptions and business barriers," arXiv preprint arXiv:1602.00173, 2016.

[4] K. Poularakis, G. Iosifidis, A. Argyriou, I. Koutsopoulos, and L. Tassiulas, "Caching and operator cooperation policies for layered video content delivery," IEEE International Conference on Computer Communications (INFOCOM), 2016.

[5] M. Gregori, J. Gómez-Vilardebò, J. Matamoros, and D. Gündüz, "Wireless content caching for small cell and D2D networks," IEEE Journal on Selected Areas in Communications, vol. PP, no. 99, pp. 1-1, 2016.

[6] S. E. Ghoreishi, V. Friderikos, D. Karamshuk, N. Sastry, and A. H. Aghvami, "Provisioning cost-effective mobile video caching," in IEEE International Conference on Communications (ICC), Kuala Lumpur, Malaysia, 2016

[7] X. Peng, J. Zhang, S. Song, and K. B. Letaief, "Cache size allocation in backhaul limited wireless networks," arXiv preprint arXiv:1602.08728, 2016.

[8] F. Alotaibi, S. Hosny, J. Tadrous, H. E. Gamal, and A. Eryilmaz, "Towards a marketplace for mobile content: Dynamic pricing and proactive caching," arXiv preprint arXiv:1511.07573, 2015.

[9] Z. Chen, Y. Liu, B. Zhou, and M. Tao, "Caching Incentive Design in Wireless D2D Networks: A Stackelberg Game Approach," in IEEE International Conference on Communications (ICC), Kuala Lumpur, Malaysia, 2016. 